OPEN ACCESS

Edited by:

Catherine Anne Kidner, University of Edinburgh, UK

Reviewed by:

Christian Schulz,

Evolution and Biodiversity of Plants,

Germany

Mark Hughes,

Royal Botanic Garden Edinburgh, UK

*Correspondence:

Stefan Wanke

stefan.wanke@tu-dresden.de

${ }^{\dagger}$ Present address:

Marie-Stéphanie Samain, Instituto de Ecología, A.C., Centro Regional del Bajio, Avenida Lázaro Cárdenas 253, Pátzcuaro, Mexico

Specialty section:

This article was submitted to Plant Evolution and Development, a section of the journal Frontiers in Plant Science

Received: 25 April 2016 Accepted: 18 July 2016 Published: 09 August 2016

Citation:

Frenzke L, Goetghebeur P, Neinhuis C, Samain M-S and

Wanke S (2016) Evolution of Epiphytism and Fruit Traits Act Unevenly on the Diversification of the

Species-Rich Genus Peperomia (Piperaceae). Front. Plant Sci. 7:1145. doi: 10.3389/fpls.2016.01145

\section{Evolution of Epiphytism and Fruit Traits Act Unevenly on the Diversification of the Species-Rich Genus Peperomia (Piperaceae)}

\author{
Lena Frenzke ${ }^{1}$, Paul Goetghebeur ${ }^{2}$, Christoph Neinhuis ${ }^{1}$, Marie-Stéphanie Samain ${ }^{2 \dagger}$ and \\ Stefan Wanke ${ }^{1 *}$
}

${ }^{1}$ Department of Biology, Institut für Botanik, Technische Universität Dresden, Dresden, Germany, ${ }^{2}$ Department of Biology, Research Group Spermatophytes, Ghent University, Gent, Belgium

The species-rich genus Peperomia (Black Pepper relatives) is the only genus among early diverging angiosperms where epiphytism evolved. The majority of fruits of Peperomia release sticky secretions or exhibit hook-shaped appendages indicative of epizoochorous dispersal, which is in contrast to other flowering plants, where epiphytes are generally characterized by fruit morphological adaptations for anemochory or endozoochory. We investigate fruit characters using Cryo-SEM. Comparative phylogenetic analyses are applied for the first time to include life form and fruit character information to study diversification in Peperomia. Likelihood ratio tests uncover correlated character evolution. We demonstrate that diversification within Peperomia is not homogenous across its phylogeny, and that net diversification rates increase by twofold within the most species-rich subgenus. In contrast to former land plant studies that provide general evidence for increased diversification in epiphytic lineages, we demonstrate that the evolution of epiphytism within Peperomia predates the diversification shift. An epiphytic-dependent diversification is only observed for the background phylogeny. An elevated frequency of life form transitions between epiphytes and terrestrials and thus evolutionary flexibility of life forms is uncovered to coincide with the diversification shift. The evolution of fruits showing dispersal related structures is key to diversification in the foreground region of the phylogeny and postdates the evolution of epiphytism. We conclude that the success of Peperomia, measured in species numbers, is likely the result of enhanced vertical and horizontal dispersal ability and life form flexibility but not the evolution of epiphytism itself.

Keywords: BaMM, BiSSE, diversification, dispersal, epiphytism, epizoochory, fruit morphology, life form

\section{INTRODUCTION}

Epiphytism evolved in various green plant lineages ranging from bryophytes and ferns to flowering plants (Gentry and Dodson, 1987). The majority of vascular epiphytes are found in the monocots and eudicots (75 and 13\%, respectively) (Zotz, 2013). Epiphytism is generally hypothesized to enhance diversification, due to the large number of microhabitats and high niche fragmentation 
(Gentry and Dodson, 1987; Benzing, 1990). Orchids, Bromeliaceae, and Gesneriaceae are well known examples of higher species richness in epiphytic flowering plant lineages, compared to terrestrial sister lineages (Gentry and Dodson, 1987; Gravendeel et al., 2004; Zotz, 2013; Givnish et al., 2015). This increased diversity has raised the question of factors shaping or contributing to the rate of diversification of epiphytic lineages. Especially in closed habitats such as tropical rainforests, the epiphytic life style provides potentially extended access to light compared to the terrestrial habitat. Epiphytism offers the possibility for occupying bark, branches and twigs that altogether provide a greater habitable surface compared to the ground level. Niche partitioning in climatic heterogeneous tree crowns furthermore is thought to maintain increased plant diversity (Gentry and Dodson, 1987; Benzing, 1990; Silvera et al., 2009). However, it is largely unclear whether morphological or physiological traits are a prerequisite for plants to become epiphytes or if character (trans) formations are a result of life form shifts and lead to diversification. Disentangling the temporal origin of traits and their impact on speciation or extinction rates is essential for understanding diversification and the underlying evolutionary processes.

With the rise of comparative evolutionary methods allowing for a trait's influence on evolutionary processes, interferences on character state-dependent diversification can be directly inferred (e.g., Maddison et al., 2007; FitzJohn, 2012; Rabosky, 2014; Rabosky et al., 2014). Furthermore, methods for analyzing character state-independent diversification dynamics on phylogenies play a central role in phylogenetic research (Rabosky et al., 2007; Alfaro et al., 2009; Rabosky, 2014; Rabosky et al., 2014).

Recent studies confirmed the hypothesis of higher diversification rates in epiphytes compared to terrestrials within Bromeliaceae (Givnish et al., 2014) and Orchids (Givnish et al., 2015), and for epiphytes compared to generalists within ferns (Feldberg et al., 2014). Moreover, these new approaches reduce the biases in the interpretation of characters as possible key innovations ( $\mathrm{Ng}$ and Smith, 2014). Key innovations represent novel characters, which are directly linked to increased diversification rates, either by reducing extinction or by accelerating speciation (Sanderson and Donoghue, 1994).

Peperomia (Piperaceae, Black Pepper relatives) is the only genus among the early diverging angiosperms ("basal angiosperms", i.e., lineages which diverged prior to monocots and eudicots) where epiphytism evolved (Isnard et al., 2012). Among flowering plants, Peperomia comprises the largest number of epiphytic species in a genus apart from some orchid genera and Tillandsia (Bromeliaceae) (Zotz, 2013) and it is listed among the ten most species-rich flowering plant genera in general (1606 species) (Frodin, 2004; Samain et al., 2009, 2011; Mathieu et al., 2011; Frenzke et al., 2015). The genus is pantropically distributed with the highest diversity in the Neotropics (Wanke et al., 2006; Smith et al., 2008; Samain et al., 2011; Pino et al., 2012), where it is a notable component of the epiphytic (Barthlott et al., 2001; Krömer et al., 2007) and terrestrial (Mathieu et al., 2011; Samain et al., 2011) flora in a wide range of vegetation types. Molecular phylogenies of
Peperomia revealed terrestrial clades to be successive sister to the remaining lineages in which epiphytism occurs at least frequently or is the predominant life form (Samain et al., 2009; Frenzke et al., 2015). The fruits (up to $3.5 \mathrm{~mm}$ in size) are small drupes consisting of a thin mesocarp, a stony endocarp and a hard testa (Johnson, 1900; Fisher, 1914; Burger, 1971). As many Peperomia species show sticky secretions on the fruit surface (Frenzke et al., 2016), epizoochorous dispersal has long been suggested by most (e.g., Dahlstedt, 1900; Ridley, 1930; Yuncker, 1958; Carlquist, 1967; Burger, 1971; Croat, 1978; Melcher et al., 2000), but not all authors (Bradley, 2002). Although information on Peperomia dispersing animals is sparse, birds are considered as vectors by Ridley (1930) and Carlquist (1967). The investigation of epizoochorous dispersal is fascinating as adhesion (including mechanical attachment mechanisms) is, with less than 5\%, sparsely represented in plants. Even more particular is the adhesion by sticky substances (Sorensen, 1986). The majority of epiphytes (84\%) propagate by tiny wind dispersed seeds called sporochores, followed by fruits showing winged or plumed seeds or adaptations to endozoochory (birds, bats) (Gentry and Dodson, 1987; Benzing, 2004). In contrast, terrestrial lineages frequently show more generalistic dispersal modes. Increasing dispersal ability and the colonization of new niches are thought to affect diversification as they act by altering gene flow ( $\mathrm{Ng}$ and Smith, 2014). Peperomia is the only genus considered as epizoochorous throughout the literature on epiphyte dispersal (Yuncker, 1958; Madison, 1979; van der Pijl, 1982; Armesto and Rozzi, 1989; Hughes et al., 1994; Benzing, 2004) and to our knowledge the only epiphytic plant lineage developing sticky fruits. The species richness, the high number of epiphytes, as well as the fruit morphological modifications indicative for epizoochorous dispersal, make Peperomia a unique case to study life form evolution, fruit morphology, and diversification in a comparative manner.

We examined Peperomia fruits by cryo-scanning electron microscopy (cryo-SEM) to uncover dispersal-related traits. A molecular phylogeny of 114 Peperomia species covering the taxonomical and life form diversity of the genus is reconstructed. Life form is assigned to 1520 species. This framework serves as basis for state-dependent and state-independent diversification analyses, ancestral character state reconstructions, as well as tests for correlated evolution. We aim to test the overarching hypothesis that evolution of fruit morphological adaptations and transition from terrestrial to epiphytic life form fostered diversification in the species-rich genus Peperomia.

\section{MATERIALS AND METHODS}

One hundred fourteen Peperomia species were examined, representing the genus' taxonomical, morphological and life form diversity (Frenzke et al., 2015). Plant material was collected in the field or taken from greenhouse collections of the Botanical Gardens of Ghent University (Belgium) and Dresden (Germany). Character scoring was completed with data from literature (e.g., Trelease and Yuncker, 1950; Yuncker, 1953, 1958, 1974; Samain et al., 2009). Successive sister lineages of Peperomia were used as 
outgroup (Piper and Saururaceae) (Wanke et al., 2007a,b; Samain et al., 2008; Naumann et al., 2011).

\section{Cryo-Scanning Electron Microscopy}

We used Cryo-SEM to observe fruit micromorphology. Fruits were regarded as fully mature when they easily detach from the spadix upon slightest contact. Cryo-SEM protocols followed Frenzke et al. (2015).

\section{Phylogenetic Analyses}

For the phylogenetic analysis based on the chloroplast trnKmat K-psbA region, we sampled 114 Peperomia species. The selection was based on recently published molecular data (Frenzke et al., 2015), to which another 24 accessions were added. However, the molecular dataset includes less species compared to Frenzke et al. (2015), because precise information on fruit characters was not available for all species. Voucher and origin, as well as Genbank accession numbers of the 24 additional accessions are provided as supporting information (Supplementary Table S1). DNA extraction protocol, PCR settings, ingredients, and concentrations, as well as purification of products followed Frenzke et al. (2015). Sequences were obtained using Macrogen Inc. or a Beckman Coulter capillary lab sequencer. Sequence data were manually edited and aligned using PhyDE $^{\circledR}$ version 0.9971 (Müller et al., 2010), excluding regions of uncertain homology prior to analyses (Supplementary Table S2). A Maximum likelihood (ML) analysis was conducted using RAxML (Stamatakis et al., 2005) applying the GTR $+\Gamma$ model and sampling 1000 rapid bootstrap replicates. The best fitting model was found using JMODELTEST (Guindon and Gascuel, 2003; Posada, 2008; Darriba et al., 2012). Bayesian Inference (BI) was performed using MRBAYES version 3.1.2 (Ronquist and Huelsenbeck, 2003) implementing four independent runs with four chains of 2,000,000 MCMC (Markov Chain Monte Carlo) generations each, saving every 200th tree. The first 300,000 generations of each run were discarded as burn-in as evaluated by TRACER (Rambaut and Drummond, 2009). FigTree version 1.4.0 (Rambaut, 2008) was used for tree editing.

The concatenated alignment of the trnK-matK-trnK-psbA dataset included 4418 characters from which 21 regions comprising 619 characters were excluded due to uncertain homology (Supplementary Table S2). Backbones of BI and ML topologies were highly congruent and supporting values for both methods are given on the ML tree (Supplementary Figure S1). The resulting majority rule (50\%) consensus tree from $\mathrm{BI}$ analysis was used for our diversification study (Figure 1).

Further analyses required ultrametric phylogenetic trees. After pruning outgroup species from the consensus tree we run the 'chronos' function (Sanderson, 2002) of the package 'ape' (Paradis et al., 2004) in R using RStudio 0.99.491 (R Studio Inc., Boston, MA, USA). We set the smoothing parameter to 0 , allowing the full range of rate variation among branches. As there are no fossils recorded for Peperomia, we decided to set the tree height to 1 to avoid temporal bias (e.g., Granados Mendoza et al., 2015). All trees were subsequently rescaled assigning branch tips to time 0 and root to time 1 .

\section{Diversification Shift Analysis}

For the detection of heterogeneity in diversification rates and to test for credible rate shift configurations, we applied Bayesian analysis of macroevolutionary mixtures (BaMM, version 2.5.0). BaMM simulates posterior distributions of rateshift configurations by 'reversible jump' Markov Chain Monte Carlo (rjMCMC) to account for rate variation through time and among lineages (Rabosky, 2014). BaMM can account for incomplete, non-random taxon sampling by allowing individual clades to have different sampling probabilities. We assigned the latest information on species richness to clades (Figure 1; Frenzke et al., 2015). The $\mathrm{R}$ function 'BaMM priors' was applied to get appropriate prior parameters based on the consensus tree. We choose a Poisson rate prior of 1.0 , proposed by the default settings in BaMM as conservative approach. We run BaMM for ten chains of 5,000,000 generations, saving every 1000. Convergence and effective sample size were tested by applying the ' $R$ ' package CODA v. 0.18-1 (Plummer et al., 2006). We discarded 10\% of the MCMC generations as burn-in prior to output analyses in R. The R package 'BaMMtools' (Rabosky et al., 2014) was used for postrun analysis of BaMM output files to identify the $95 \%$ credible set of shift configurations and to trace clade-specific diversification rates through times.

\section{Character Coding and Rationale}

Fruit morphological characters and life form were scored (Supplementary Table S3). Life form was coded as terrestrial (0) or epiphytic (1), with geophytic and lithophytic considered as terrestrial (Zotz, 2013). We scored the general presence of fruit's adhesiveness (manually tested) as absent (0) and present (1). It is, however, unclear if the fruit stickiness originates from anatomically homologs structures. Fruit adhesiveness is caused by epidermal structures, which may be secretive papillate cells (Peperomia subgenera Micropiper, Oxyrhynchum, Leptorhynchum), or the so-called pseudocupula (Peperomia subg. Pseudocupula). The latter was reported as an epidermal structure covering the fruit base (Dahlstedt, 1900). In addition, punctual sticky secretion is observed as an intermediate state in some species of Peperomia subg. Oxyrhynchum. In those cases, stickiness could be verified, but the secreting structures were neither pseudocupula nor papillate cells. Fruit trichomes of $P$. hispidula and $P$. hispiduliformis were not regarded as adhesive because they are not glandular (Martínez-Colín et al., 2006). The pseudopedicel represents a stalk-like outgrowth of the rachis and has been regarded as a dispersal-related structure that develops during fruit maturation (Dahlstedt, 1900; Yuncker, 1958). It was coded as absent (0) or present (1). The fruit apex is potentially relevant for dispersal as it forms hook-shaped appendages in some species and was coded as being absent (0) or present (1).

For the analyses of fruit character evolution we pruned outgroup species, as character states were not applicable. Drupes of the genus Piper differ notably in size, color and amount of fleshy mesocarp and show no obvious structures for mechanical attachment and external dispersal (Yuncker, 1958) (Rauh, 1950). The analysis of life form is based on 1520 Peperomia species and additionally includes outgroup lineages (Isnard et al., 2012). 


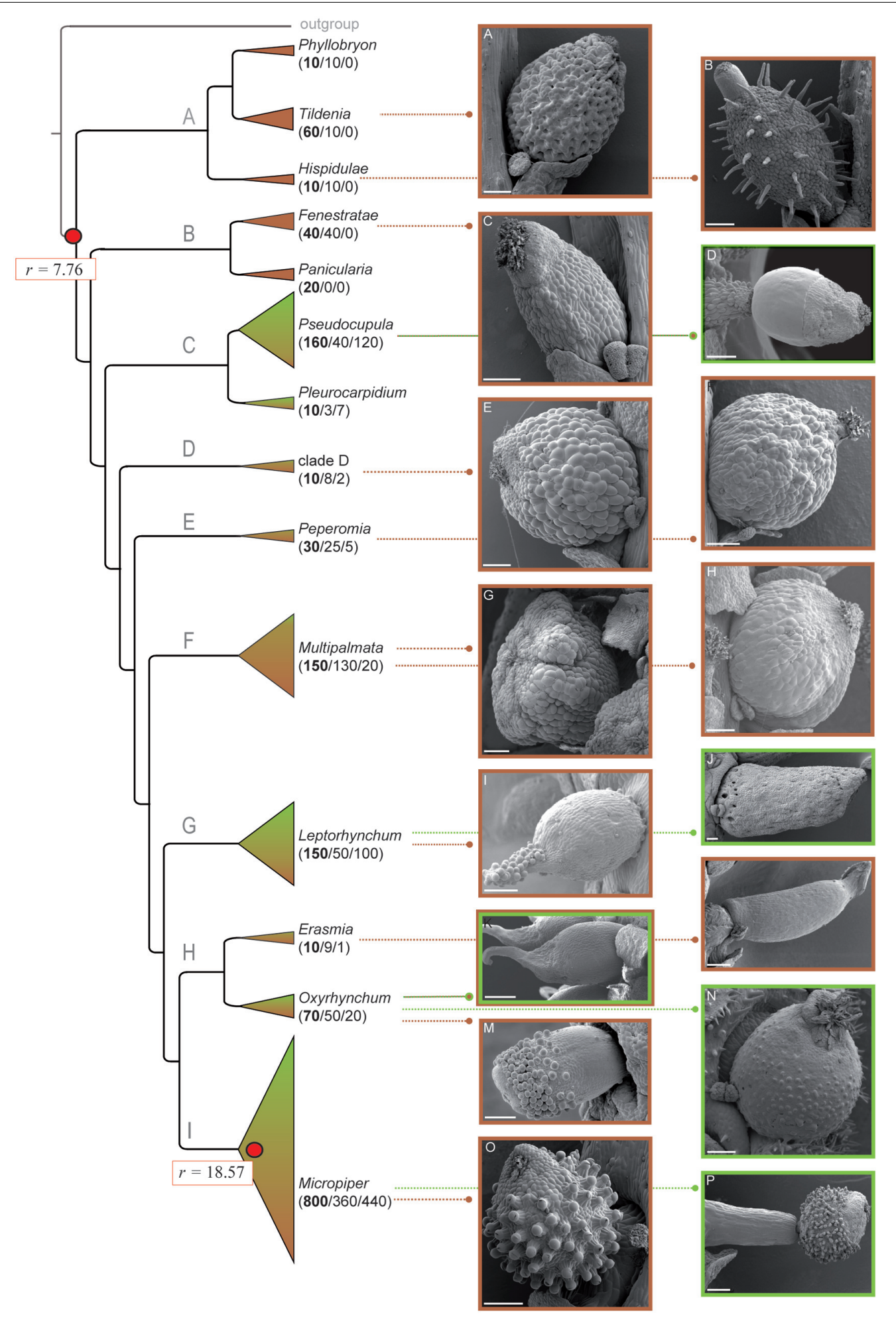

FIGURE 1 | Peperomia fruit characters and life form distribution. Pointed lines lead to SEM images of fruit phenotypes representative for each clade. Subgenera are named according to Frenzke et al. (2015). Color coding of picture frames and triangles of clades of the phylogenetic tree indicate the different life forms (brown-terrestrial; green-epiphytic). Shading of these clades is proportional to the respective species numbers being characterized by either terrestrial or epiphytic life form. Numbers in brackets refer to the estimated total number of species of each clade, the number of terrestrial species, and to the number of epiphytic species. The background rate for the entire tree, as well as the specific net diversification rate for the foreground clade are marked with red circles. (A) P. bracteata, (B) P. hispidula, (C) P. dolabriformis, (D) P. dahlstedtii, (E) P. maypurensis, (F) P. pellucida, (G) P. procumbens, (H) P. arifolia, (I) P. species, (J) P. crassicaulis, (K) P. obtusifolia var. emarginata, (L) P. lancifolia, (M) P. pernambucensis, (N) P. hirta, (O) P. inaequalifolia, (P) P. bicolor. Scale bars 200 m. 


\section{Binary State Speciation and Extinction Analyses}

We used BiSSE (Maddison et al., 2007) as implemented in the R package 'diversitree' (FitzJohn, 2012) to estimate evolutionary parameters. BiSSE simultaneously estimates diversification and transition rates without requiring ancestral state reconstructions (ASRs). The BiSSE model assumes that speciation and extinction follow a birth-death process, where rates depend on a certain character state. It thus adds character-dependent parameters to the commonly used Markov models of character evolution (Mk models; Pagel, 1994; O’Meara, 2012). All BiSSE calculations were performed on the consensus tree obtained by BI. We applied the 'skeletal tree' approach with a sampling frequency of 0.1 for the fruit morphological characters. For some species, fruit characters could not be studied and information on fruit characters in the literature is often vague. We assume our sampling being representative as species of every clade of the phylogeny were included in the analysis. Within clades a random subset of one to 20 species was sampled in relation to clade size. Information on life forms of all known Peperomia species was included by applying the 'unresolved tree approach' for this character where life form distributions were assigned to terminal clades (Figure 1, numbers in brackets). Twelve different evolutionary models for life form and fruit functional characters were compared, testing character-associated parameters. The full BiSSE model includes six parameters, where speciation $(\lambda)$, extinction $(\mu)$, and transition $(q)$ rates are calculated for each of the two character states $(0,1)$. We compared the full model $\left(\lambda_{0}, \lambda_{1}, \mu_{0}, \mu_{1}, q_{01}, q_{10}\right)$ to constrained models with speciation, extinction, and transition rates subsequently set to equal or zero $\left(\lambda_{0}=0 ; \lambda_{1}=0 ; \lambda_{0}=\lambda_{1} ; \mu_{0}=0\right.$; $\left.\mu_{1}=0 ; \mu_{0}=\mu_{1}, q_{01}=0 ; q_{10}=0 ; q_{01}=q_{10}\right)$. We tested the $\mathrm{Mk} 2$ model as alternative hypothesis to explain character distributions independent from state-specific speciation and extinction. The Mk2 model (4-parameter model) was treated as a constrained model of the full model with speciation and extinction rates set equal for both states and transition rates varied freely $\left(\lambda_{0}=\lambda_{1}, \mu_{0}=\mu_{1}\right)$. To account for heterogeneity in diversification rates related to life form and fruit character evolution, we furthermore applied the function 'bisse.split'. 'Bisse.split' uses an expanded 12-parameter BiSSE model where parameter sets are estimated independently for partitions of the phylogeny. We chose to split the phylogeny at the point that is identified by BaMM as the most probable shift of the $95 \%$ credible set of shift configurations $(P=0.65$, Supplementary Figure S2A). Hence, parameters were estimated separately for the background $\left(\lambda_{0.1}, \lambda_{1.1}, \mu_{0.1}\right.$, $\left.\mu_{1.1}, q_{01.1}, q_{10.1}\right)$ and the foreground partition $\left(\lambda_{0.2}, \lambda_{1.2}\right.$, $\left.\mu_{0.2}, \mu_{1.2}, q_{01.2}, q_{10.2}\right)$. In a first step, model parameters were optimized with ML searches on our consensus tree obtained by BI. To test which model explains our data best, and thus how life form and dispersal related fruit characters have influenced diversification we compared the log likelihoods (lnLs) of all models using the AIC (Maddison et al., 2007) and related Akaike weights $\left(w_{\mathrm{i}}\right)$ (Burnham and Anderson, 2002).
To account for parameter uncertainty we performed Bayesian MCMC using slice sampling (Neal, 2003) under the best fitting model. An exponential prior with rate $1 /(2 r)$ was used for MCMC simulations. $r$ is the character-independent diversification rate obtained by BiSSE ML (Maximum Likelihood) estimation. A preliminary set of 1,000 MCMC steps was run to obtain $a$ priori estimates for the tuning parameter $w(0.95$ density of $\mathrm{PP})$ (Neal, 2003). Final MCMC chains (with $w$ specified) were run for 20,000 iterations to obtain posterior distributions of evolutionary parameters. Although chains converged rapidly, a burn-in fraction of 25\% MCMC steps was discarded (conservative approach). Post burn-in MCMC samples were tested for effective sample size above 200 using the MCMC output analysis and diagnosis package 'coda' (Plummer et al., 2006) implemented in R. 95\% Credibility Intervals (CIs) of posterior distributions are provided for each parameter set. We summed proportions of post burn-in samples with higher rates for state 1 compared to state 0 and treated them as PP. We considered PP $\geq 0.95$ as support for significant higher rates.

Rabosky and Goldberg (2015) recently showed that if a tree evolves under a heterogeneous branching process that is completely independent from the evolution of the character to be investigated; SSE models [state speciation and extinction models; see Rabosky and Goldberg (2015)] will, in many cases, return high support for a model of trait-dependent diversification. By applying BaMM as cross-validation to test for model violation and by applying a partitioned SSE model to account for diversification rate heterogeneity in our phylogeny, we address for possible inadequacy reported by Rabosky and Goldberg (2015). ASR was performed under the best fitting BiSSE (Binary State Speciation and Extinction) model to account for the influence of life forms and fruit character states on the diversification of Peperomia. We applied the BiSSE marginal ASR as implemented in the R-package 'diversitree' (FitzJohn, 2012). To integrate parameter uncertainty in ancestral character state reconstructions we run 1,000 MCMC iterations. The same priors as for BiSSE MCMC analysis were applied to start ASR-MCMC chains. Mean values of proportional likelihoods were calculated after discarding a burn-in of 25\% from the MCMC samples and ancestral state probabilities were mapped onto main nodes of the consensus tree with a particular state being most likely at a significance ratio of 0.86 (7.4:1) or above (Edwards, 1972; Schluter et al., 1997).

\section{Tests for Correlated Evolution}

Correlated evolution among fruit characters and life form was tested using Pagel's correlation analysis (Pagel, 1994) implemented in the Correl Package version 0.1 of Mesquite (Midford and Maddison, 2006). We run this analysis as an exploratory analysis without specifying a priori assumptions on the evolutionary association for all pairwise combinations. Pagel's approach uses the LRT statistic to discriminate between two models. One model assumes for correlated state changes of the two tested characters and consequently applies eight parameters. The second model represents a constrained version of the eight-parameter model and describes the transition 
between character states independently from transitions of the second character using four parameters. We run Pagel's test on a subset of ten BI trees applying $30 \mathrm{ML}$ iterations. Simulations were computationally expensive and did not affect $P$-values. We therefore decided to run 1000 MCMC iterations on only one tree of the subset for each character combination. For comparison of model fitting, AIC and $w_{\mathrm{i}}$ were calculated as described above. Accessions with unknown or missing character states were excluded prior to analyses.

\section{RESULTS}

A-well resolved and supported phylogeny based on 4118 bp alignment characters resulted in nine main lineages (Figures 1 A-I; Supplementary Figure S1), corresponding to subgenera and unnamed clades (Figure 1, Supplementary Figure S1, Frenzke et al., 2015). Lineages A and B comprise exclusively terrestrial species, lacking fruit characteristics such as pseudopedicel, sticky secretions, and beaks (Figures 1A-C). Lineage C includes 18\% (127 species) of all epiphytes. Fruits of Peperomia subg. Pseudocupula are characterized by sticky secretions on the fruit surface, the so-called pseudocupula (Figure 1D) (Samain et al., 2009; Frenzke et al., 2015). More than 50\% of the sampled Peperomia subg. Pseudocupula species exhibit a pseudopedicel, but rarely show apical beaks. The species-poor Peperomia subg. Pleurocarpidium being sister to Peperomia subg. Pseudocupula shows none of these structures. The unnamed clade D and Peperomia subg. Peperomia harbor predominantly terrestrial species and lack sticky secretions, pseudopedicel, as well as beaks (Figures 1E,F). Peperomia subg. Multipalmata as sister to the remaining Peperomia lineages is a species-rich group comprising $18 \%$ of all terrestrial Peperomia species. Diversity of fruit shapes is high in this subgenus but no means for external dispersal are observed (Figures 1G,H). The species-rich Peperomia subg. Leptorhynchum comprises one third terrestrial and two thirds epiphytic species. Species of this clade are morphological diverse with respect to fruit shapes and structures. They show sticky secretions, as well as beaks or hooks of varying length (Figures 1I,J). The fruits show a sessile or subsessile attachment without pseudopedicel formation. Lineage $\mathrm{H}$ includes the two smaller subgenera Erasmia and Oxyrhynchum (Figure 1). The mainly terrestrial Peperomia subg. Erasmia is characterized by elongated fruits with beaks and pseudopedicel formation, but no sticky secretions are observed (Figure 1L). Peperomia subg. Oxyrhynchum harbors morphologically diverse fruits with beaks or hooks and sticky secretions (Figures $1 \mathbf{K}-\mathbf{N}$ ). The most species-rich Peperomia subg. Micropiper (800 species) includes similar proportions of terrestrial and epiphytic species (Figure 1). In contrast to most other Peperomia lineages, all fruits of subg. Micropiper are characterized by sticky papillae and a pseudopedicel, and do often show a beaked apex as well (Figures 10,P).

\section{Diversification within Peperomia}

Bayesian analysis of macroevolutionary mixture Markov Chain Monte Carlo runs converged rapidly and our burn-in fraction of
0.1 was proved to be sufficient by CODA MCMC output analysis. Effective sample sizes were much greater than 200 with 2158 for the number of shift events along the phylogeny and 1525 for the lnL. 'BaMMtools' reveal our Peperomia phylogeny to violate a single regime birth-death model. The $95 \%$ credible set of shift configurations indicates rate heterogeneity within Peperomia, due to a single diversification shift (Supplementary Figure S2). The mean net diversification rate of the phylogeny is estimated with $r=7.76$ (Supplementary Figure S2B). Frequently sampled shifts of increased net diversification rate are found between time 0.28 and 0.40 located within subgenus Micropiper (Figure 2, Supplementary Figure S2B). The average net diversification rate beyond this shift ( $r=18.57$ ) exceeds twice the mean background rate for the entire tree. Clade specific diversification rates are estimated with mean values between 6.29 and 7.07 for all Peperomia subgenera except for subgenus Micropiper ( $r=15.91$; Supplementary Figures S2A,B).

\section{Character-Dependent Diversification}

Our character-dependent diversification analyses shows that the full BiSSE split model, which estimates rates of speciation, extinction and character changes independently for the foreground and the background phylogeny, provides significant better fit for all examined traits than all other models (Supplementary Table S4).

Our BiSSE MCMC analyses reveal epiphytic lineages in the background region to diversify twice as fast as the terrestrial ones (Table 1; Figure 3A). Transitions from epiphytes to terrestrials generally occur at higher rates than in the reverse direction, however, being significant in the background lineages only (Tables 1 and 2; Figure 3A). Diversification and transition rates for both epiphytes and terrestrials increase considerably after the diversification shift (Table 1; Figure 3A). However, no differences are identified between both character states in the foreground clade as indicated by largely overlapping 95\% credible intervals (CIs; Tables 1 and 2; Figure 3A).

Our results inferred generally higher diversification rates for species with sticky fruits (Table 1; Figure 3B). For this character state foreground diversification rates are found to be significantly higher (no overlapping CIs) (Table 2; Figure 3B). Transition rates in both directions are found to be generally low in the background phylogeny, however, with significantly lower probability for the gain of fruit stickiness (Table 1; Figure 3B).

Markov Chain Monte Carlo analyses under the full BiSSE split model show that species with sessile fruits diversify significantly faster than those possessing a pseudopedicel in the background phylogeny. However, the reverse is found for the foreground clade where pseudopedicellate species diversify at significantly higher rates (Table 1; Figure 3C). Largely overlapping posterior distributions in both background and foreground partitions indicate no differences in transition rates between both character states (Table 2; Figure 3C). Diversification rates of species with a fruit beak are generally higher than those lacking this structure, but this is only significant in the foreground partition (Tables 1 and 2; Figure 3D). The evolution of the beak was recovered with significantly higher loss than gain rates across the entire phylogeny (Tables 1 and 2; Figure 3D). 


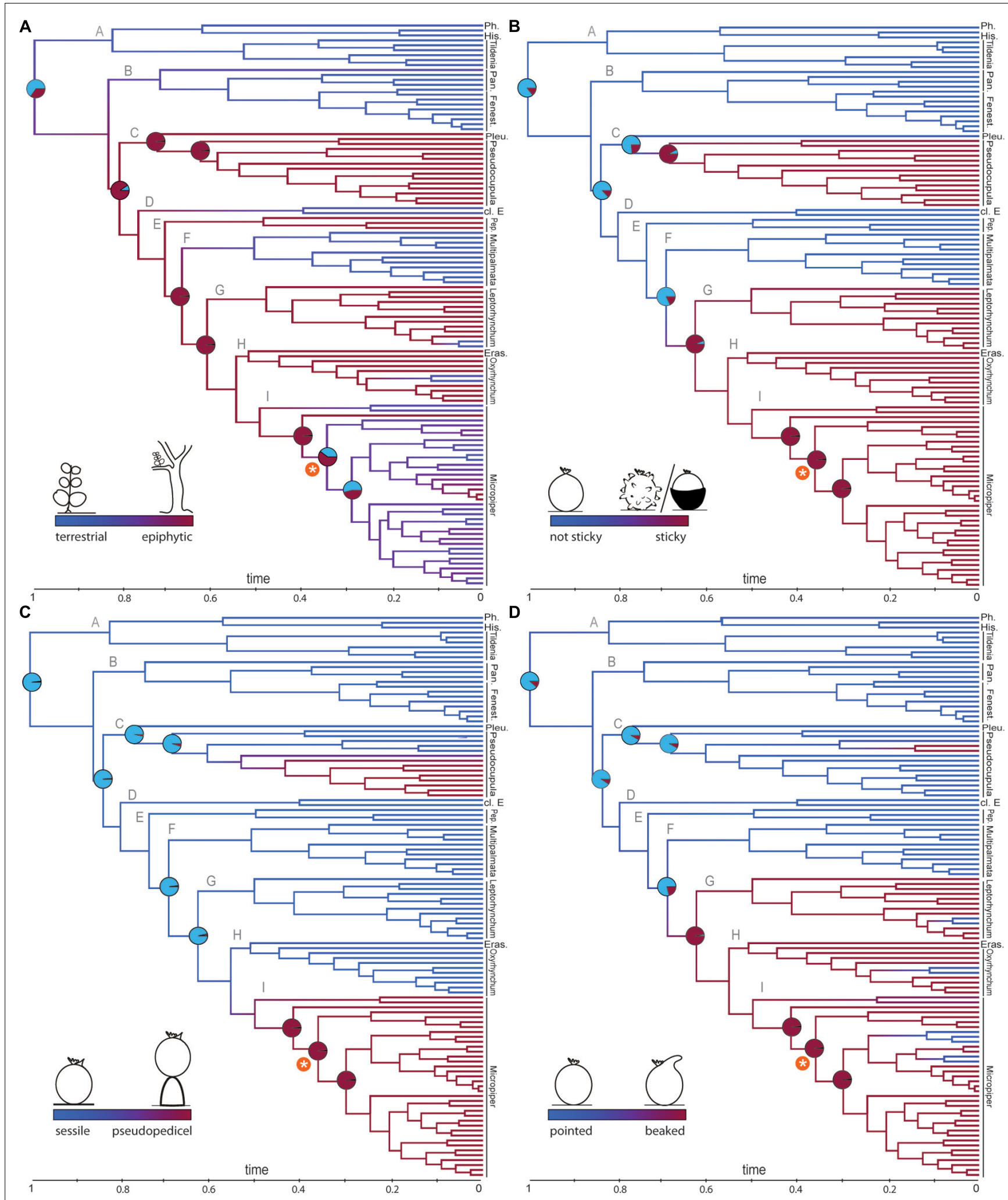

FIGURE 2 | Reconstruction of fruit traits and life form. Ancestral state reconstruction (ASR) using the BiSSE model is shown on the majority rule (50\%) consensus tree obtained from Bl analysis. (A) Epiphytism evolved before the diversification shift, (B-D) Studied fruit characters each originated twice along the phylogeny. Branch colors refer to state probabilities. Pie charts illustrate character state probabilities for main nodes. The asterisk marks the diversification shift within subg. Micropiper. 


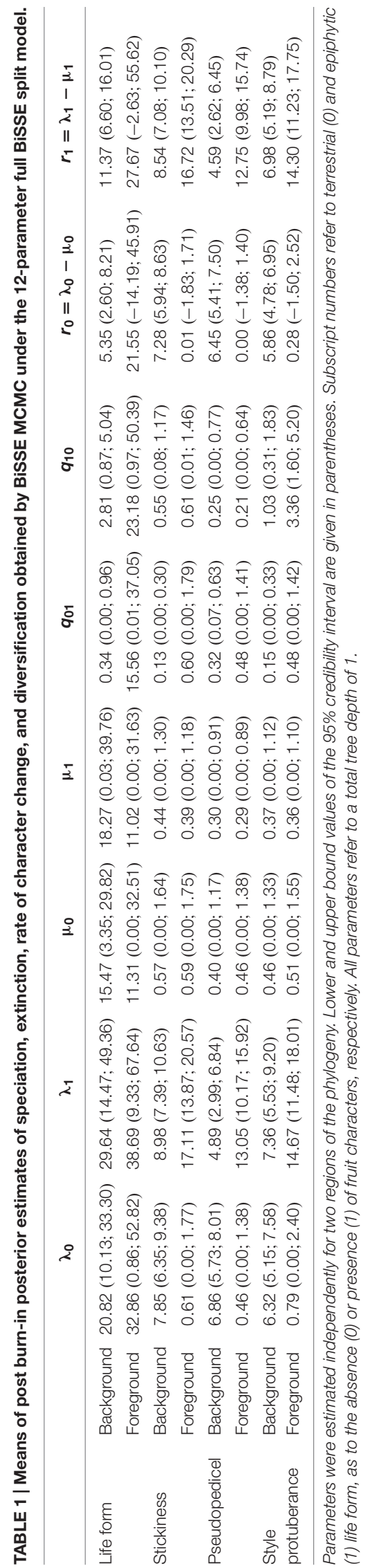

\section{Ancestral Character States}

Ancestral character state reconstructions under the full BiSSE split model inferred the most recent common ancestor (MRCA) of Peperomia to be most likely terrestrial $(P=0.73$, Figure 2A). An epiphytic MRCA is reconstructed for the node of lineage $C$ to all remaining lineages. At the shift of increased diversification rate an ambiguous reconstruction with respect to terrestrial/epiphytic is reconstructed (Figure 2A). Within the foreground clade, frequent reversals to a terrestrial life form are observed (Figure 2A).

The MRCA of the genus is reconstructed to lack sticky fruits (Figure 2B; $P=0.87$ ). Sticky fruits are recovered with two independent origins, predating the diversification shift (Figure 2B). Reversals to fruits lacking sticky structures were not recovered.

A sessile attachment of fruits is reconstructed as ancestral state for the MRCA of Peperomia $(P=0.97$; Figure $2 \mathrm{C}$ ), while the pseudopedicel evolved twice independently, once within subg. Pseudocupula and once within subg. Micropiper (Figure 2C). Reverse transitions to fruits without pedicels are not discovered.

Pointed fruits are the most likely ancestral state for Peperomia $(P=0.88$, Figure 2D) and an apical beak-shaped protuberance originated at least twice. Frequent reversals to fruits lacking the apical beak are observed within the subgenera Leptorhynchum, Oxyrhynchum and Micropiper (Figure 2D).

\section{Correlated Evolution}

The dependent model (four-parameter) is preferred over the model of independent evolution (eight-parameter) due to a higher likelihood and higher $w_{\mathrm{i}}$ for all tested character combinations. Correlated evolution of life form and fruit stickiness is statistically supported (Table $3, w_{\mathrm{i}}>0.99$ ) in all trees. In addition, the dependent parameter model favored the explanation of epiphytic evolution in association with fruit beaks in $90 \%$ of the analyzed trees (Table 3). Testing all fruit character combinations, only fruit beak and pseudopedicel evolved in correlation (Table 3, nine trees, $w_{\mathrm{i}}>0.96$ ). Evolutionary changes in life form are unrelated to pseudopedicel evolution (Table 3).

\section{DISCUSSION}

Here we present the first study on diversification of the genus Peperomia that belongs to the top 10 most species-rich genera of angiosperms (Frodin, 2004). Former studies addressing the evolution of the genus mainly focused on systematics (e.g., Samain et al., 2007, 2011; Mathieu et al., 2008; Frenzke et al., 2015), biogeography (Symmank et al., 2008, 2011), relationships among clades (Wanke et al., 2006; Samain et al., 2009) or life and growth forms in a broader context (Isnard et al., 2012). These studies form the basis to combine knowledge on characteristic structures and molecular phylogenetics in comparative analyses to figure out whether and how life forms and fruit structures influence diversification within Peperomia.

We demonstrate that diversification within Peperomia is not homogenous across its evolution and more complex than initially thought. Given that the full BiSSE split model best fits our data, 

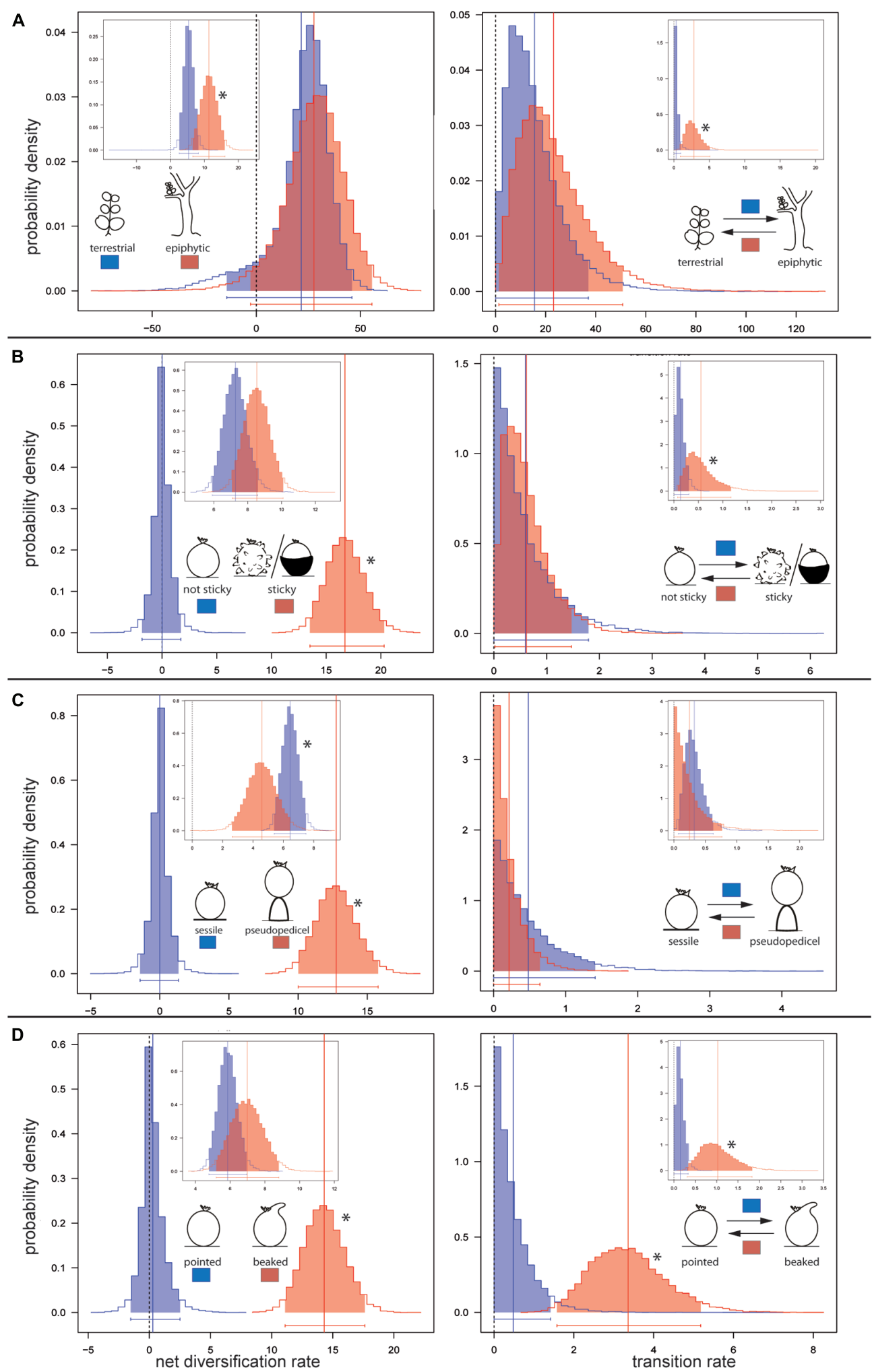

FIGURE 3 | Posterior distributions of diversification rate (left) and character transition rate (right). Rates were estimated by BiSSE MCMC under the 12-parameter full BiSSE split model. Main profile plots show foreground parameter estimates. Inbox plots show respective estimations for the background phylogeny. Rate estimations are shown for (A) terrestrial and epiphytic Peperomia lineages; (B) for lineages lacking and those possessing sticky fruits; (C) for lineages without pseudopedicel and with pseudopedicel formation, and (D) for lineages showing pointed fruits and those with beaked fruits. * marks significantly higher rates for one state over another. 
TABLE 2 | Differences of transition and diversification rates inferred from MCMC sample proportions.

\begin{tabular}{|c|c|c|c|c|c|c|c|c|}
\hline & \multicolumn{2}{|c|}{ Life form } & \multicolumn{2}{|c|}{ Stickiness } & \multicolumn{2}{|c|}{ Pseudopedicel } & \multicolumn{2}{|c|}{ Beak } \\
\hline & Background & Foreground & Background & Foreground & Background & Foreground & Background & Foreground \\
\hline $\operatorname{PP}\left(q_{10}>q_{01}\right)$ & $0.99 *$ & 0.73 & $0.95^{*}$ & 0.56 & 0.30 & 0.31 & $>0.99 *$ & $>0.99 *$ \\
\hline $\mathrm{PP}\left(r_{1}>r_{0}\right)$ & $0.98^{*}$ & 0.57 & 0.88 & $>0.99 *$ & 0.05 & $>0.99 *$ & 0.84 & $>0.99 *$ \\
\hline
\end{tabular}

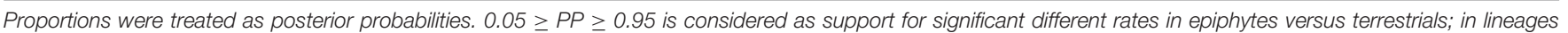

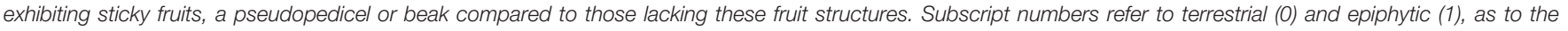
absence (0) or presence (1) of fruit characters. * marks significant differences.

TABLE 3 | Means of log likelihood (InL) and Akaike information criterion (AIC) of the two tested models for all possible pairs of characters.

\begin{tabular}{|c|c|c|c|c|c|c|c|}
\hline \multirow[t]{2}{*}{ Character combination } & \multicolumn{2}{|c|}{ 4-parameter model } & \multicolumn{2}{|c|}{ 8-parameter model } & \multirow[t]{2}{*}{$\Delta \mathrm{AIC}$} & \multirow[t]{2}{*}{$w_{\mathbf{i}}$} & \multirow{2}{*}{$\begin{array}{c}\text { \# trees supporting } \\
\text { 8-par. Model }\end{array}$} \\
\hline & $\ln L$ & AIC & $\ln L$ & AIC & & & \\
\hline epiphytism / fruit stickiness & -84.18 & 176.37 & -71.48 & 158.96 & 17.41 & $>0.99$ & $10 / 10$ \\
\hline epiphytism / pseudopedicel & -97.24 & 202.47 & -90.25 & 196.49 & 5.98 & $>0.97$ & $3 / 10$ \\
\hline epiphytism / fruit beak & -114.73 & 237.47 & -106.09 & 228.18 & 9.92 & $>0.98$ & $9 / 10$ \\
\hline fruit stickiness / pseudopedicel & -63.20 & 134.41 & -57.00 & 130.00 & 4.41 & $>0.96$ & $2 / 10$ \\
\hline fruit stickiness / fruit beak & -81.29 & 170.58 & -73.79 & 163.59 & 6.99 & $>0.97$ & $6 / 10$ \\
\hline pseudopedicel / fruit beak & -93.76 & 195.51 & -85.25 & 186.49 & 9.02 & $>0.96$ & $9 / 10$ \\
\hline
\end{tabular}

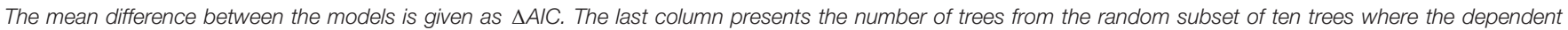
8-parameter model was significantly supported over the independent 4-parameter model by Akaike weights $\left(w_{\mathrm{i}}\right)$ above 0.95.

we confirm that life form and fruit characters did not evolve in a constant manner throughout Peperomia. Although the shift to epiphytism was likely a driver of diversification, the increase in diversification of the most species-rich subgenus rather correlates with fruit characters. We rather hypothesize that evolutionary flexibility of life forms coincides with the diversification shift.

As already indicated in the methods section, SSE approaches may infer associations of neutral traits and diversification especially if only a single diversification shift is discovered (Rabosky and Goldberg, 2015) and thus results have to be interpreted carefully. However, we choose a cross-validation by applying both BaMM and BiSSE approaches to reduce false positive results. We are furthermore aware that our study provides a first glimpse because diversification might be influenced by a complex interplay of ecological and morphological features. Additionally, conclusions on extinction rates should be drawn carefully in the absence of a fossil record (Rabosky, 2010).

\section{Life Form and Diversification}

Epiphytism is generally regarded as enhancer of diversification (Gentry and Dodson, 1987; Benzing, 2004). For epiphytic bromeliad subfamilies, Givnish et al. (2014) uncovered high rates of net diversification. A similar finding was uncovered for orchids, where the epiphytic habit appears to have accelerated net diversification rates (Givnish et al., 2015). In Peperomia, the epiphytic life form was driving diversification before the rate shift (Figure 3A). Life form associated rate differences disappear after the diversification shift, where speciation and extinction rates of epiphytes and terrestrials are found to be similar. Hence, epiphytism is not linked to the diversification shift although our analyses indicate a gradual evolution to epiphytism in background lineages. After the diversification shift we observe a high frequency of reversals and equal transition rates between epiphytes and terrestrials. This is in contrast to other flowering plant lineages where reversals from an epiphytic to terrestrial life form are uncommon (Crayn et al., 2004; Gravendeel et al., 2004; Givnish et al., 2014). Although epiphytism has been shown to be a result of a suite of key innovations (Gravendeel et al., 2004), we rather hypothesize that the terrestrial ancestor of Peperomia already showed traits potentially facilitating epiphytic life such as succulence or CAM metabolism.

\section{Fruit Characters and Diversification}

In addition to the evolution of epiphytism, Peperomia is also the only lineage among the Magnoliids that includes species with sticky fruits. Diversity of fruit morphology such as sticky means, appendages like hooks or beaks, and/or the pseudopedicel are characteristic for the majority of Peperomia species. Fruits lacking these structures are ancestral in Peperomia and mark the early diverging clades Phyllobryon, Hispidulae, Tildenia, Panicularia, and Fenestratae, as well as the later diverging lineages Multipalmata, Peperomia, and clade D (Figure 2). It is noteworthy that fruit stickiness and the pseudopedicel originated repeatedly and were not lost anymore in contrast to epiphytism. The irreversibility of fruit morphological changes including the development of new structures or fundamental reorganization seems to be a common finding across angiosperms (Beaulieu and Donoghue, 2013; Givnish et al., 2014). According to our reconstructions all studied fruit traits originated in the same clades (clade C and sister lineage of clade F, Figure 2). Most of the species of Peperomia subg. Micropiper show sticky secretions, apical beaks, and develop a pseudopedicel. Likewise, we find the same characters originating within Peperomia subg. 
Pseudocupula. Hence, these traits represent interesting cases of apparent convergence and are seemingly the result of similar adaptive forces acting on Peperomia fruit evolution within these two groups. According to BiSSE analyses (Figure 3), all examined fruit characters are positively associated with diversification across Peperomia evolution in the foreground clade. Although environmental changes involve the creation of key opportunities, plants must be able to access the new niche space. Furthermore, increased ability to disperse is prone to considerably affect diversification (Ng and Smith, 2014) and recent comparative studies provide evidence for fruit types and structures to influence diversification (Beaulieu and Donoghue, 2013). In Peperomia, the fruit type is conserved, i.e., the drupe, and the fruit structures studied here are suitable means for efficient dispersal. Hence, we are confident that the evolution of the examined fruit traits reflects the transition from abiotic to biotic dispersal within Peperomia and increased diversification in the foreground clade.

Our correlation analysis reveals that directional changes to epiphytism correlate with transitions to sticky fruits. However, it is important to note that according to our reconstructions, fruit traits have not been a prerequisite for the evolution of epiphytism. Moreover, we provide evidence that the ability to stick to potential vectors and the substrate has been beneficial for epiphytic as well as secondary terrestrial species.

\section{Evolutionary Consequences for Dispersal}

Peperomia species with different fruit structures can be assumed to be "adhesively dispersed" according to dispersal type definitions (Willson and Traveset, 1990). Sorensen (1986) and Bremer and Eriksson (1992) stated that few structural changes may be required to change, e.g., from abiotic to biotic dispersal. The general appearance of Peperomia fruits with a stony endocarp and the lack of visual attractiveness furthermore support the assumption of undirected, passive dispersal (van der Pijl, 1982; Dahlstedt, 1900; Kubitzki et al., 1993) by, e.g., birds (Ridley, 1930; Carlquist, 1967).

Fruits with the ability to attach to vectors are often considered key to long distance dispersal (LDD), explaining isolated populations (Sorensen, 1986; Baldwin and Wagner, 2010). The positive effect of fruit stickiness on horizontal and vertical dispersability was already stated for Peperomia by Burger in 1971: “[...] This character which allows peperomias to reach high tree-tops has resulted in many geographically widespread species". On the one hand, the only two subgenera Micropiper and Pseudocupula showing pantropical distribution that also includes remote islands provide support that Peperomia species with sticky fruits have access to an extended geographic range. The interoceanic long-distance dispersal is one of the longest

\section{REFERENCES}

Alfaro, M. E., Santini, F., Brock, C., Alamillo, H., Dornburg, A., Rabosky, D. L., et al. (2009). Nine exceptional radiations plus high turnover explain species diversity in jawed vertebrates. Proc. Nat. Acad. Sci. U.S.A. 106, 13410-13414. doi: $10.1073 /$ pnas.0811087106 ever reported for angiosperms (Valdebenito et al., 1990, 1992). On the other hand, the lack of functional adaptations to LDD has previously been reported as a reason for the restricted distribution of clades such as Tildenia (Symmank et al., 2011).

\section{AUTHOR CONTRIBUTIONS}

LF, M-SS, and SW conceived and designed the study. LF performed the analyses. PG, M-SS, CN, and SW contributed to data acquisition and interpretation. LF wrote the manuscript with the help of M-SS and SW. All authors contributed to the discussion, revised, and approved the final manuscript.

\section{FUNDING}

The study has been supported by the German Science Foundation (DFG) research projects DFG NE681/5-1, DFG NE681/5-2, DFG NE 681/11-1, the Research Foundation Flanders (FWOVlaanderen) research project FWO G.0172.07 and FWO travel grants to M-SS, and the Research Group Spermatophytes, Ghent University. Fieldwork was conducted with funding obtained from the German Academic Exchange Service (DAAD PPP Colombia and China). LF thanks the FAZIT foundation, the Dresden University of Technology and the Graduate Academy of the Dresden University of Technology for financial support.

\section{ACKNOWLEDGMENTS}

We thank Willow Zuchowski and David H. Benzing for sharing their knowledge on Peperomia fruit dispersal. Alexander Gamisch provided helpful advice using 'diversitree'. We thank the R community, especially the 'R-sig-phylo' list, for helpful answers and discussions on comparative phylogenetic analyses. Markus Günther is gratefully acknowledged for lab assistance. Field work was conducted under the following collection permit numbers (Bolivia, MDRAy-MA-VBRFMADGBAP-UAVPS N046/08; Costa Rica, 020-2012-SINAC; Mexico, SGPA/DGGFS/712/1397/07; and Peru, 009-2009-AGDGFFSDGEFFS). Thanks are due to the staff of the Botanical Gardens of Ghent University and Dresden for the cultivation of our Peperomia collection.

\section{SUPPLEMENTARY MATERIAL}

The Supplementary Material for this article can be found online at: http://journal.frontiersin.org/article/10.3389/fpls.2016.01145

Armesto, J. J., and Rozzi, R. (1989). Seed dispersal syndromes in the rain forest of Chiloé, evidence for the importance of biotic dispersal in a temperate rain forest. J. Biogeogr. 16, 219-226. doi: 10.2307/ 2845258

Baldwin, B. G., and Wagner, W. L. (2010). Hawaiian angiosperm radiations of North American origin. Ann. Bot. 105, 849-879. doi: 10.1093/aob/mcq052 
Barthlott, W., Schmit-Neuerburg, V., Nieder, J., and Engwald, S. (2001). Diversity and abundance of vascular epiphytes: a comparison of secondary vegetation and primary montane rain forest in the Venezuelan Andes. Plant Ecol. 152, 145-156. doi: 10.1023/A:1011483901452

Beaulieu, J. M., and Donoghue, M. J. (2013). Fruit evolution and diversification in campanulid angiosperms. Evolution 67, 3132-3144. doi: 10.1111/evo. 12180

Benzing, D. H. (1990). Vascular Epiphytes: General Biology and Related Biota. Cambridge: Cambridge University Press.

Benzing, D. H. (2004). "Vascular epiphytes", in Forest Canopies, eds M. D. Lowman and H. B. Rinker (London: Elsevier Academic Press), 175-211.

Bradley, U. (2002). Biogeography and Speciation of the Genus Peperomia Ruiz \& Pavón in Eastern Polynesia. [dissertation]. Trinity College, Dublin.

Bremer, B., and Eriksson, O. (1992). Evolution of fruit characters and dispersal modes in the tropical family Rubiaceae. Biol. J. Linn. Soc. 47, 79-95. doi: 10.1111/j.1095-8312.1992.tb00657.x

Burger, W. (1971). Flora costaricensis, family 41, piperaceae. Field. Bot. 35, 5-79.

Burnham, K. P., and Anderson, D. R. (2002). Model Selection and Multimodel Inference: A Practical Information-Theoretic Approach. New York, NY: Springer.

Carlquist, S. (1967). The biota of long-distance dispersal. V. Plant dispersal to Pacific Islands. Bull. Torrey Bot. Club 94, 129-162.

Crayn, D. M., Winter, K., and Smith, J. A. C. (2004). Multiple origins of crassulacean acid metabolism and the epiphytic habit in the Neotropical family Bromeliaceae. Proc. Nat. Acad. Sci. U.S.A. 101, 3703-3708. doi: 10.1073/pnas.0400366101

Croat, T. B. (1978). Flora of Barro Colorado Island. Stanford, CA: Stanford University Press.

Dahlstedt, H. (1900). Studien Über Süd- Und Central-Amerikanische Peperomien Mit Besonderer Berücksichtigung der Brasilianischen Sippen. Stockholm: PA Norsedt \& Söner.

Darriba, D., Taboada, G. L., Doallo, R., and Posada, D. (2012). jModelTest 2: more models, new heuristics and parallel computing. Nat. Methods 9:772. doi: 10.1038/nmeth.2109

Edwards, A. W. F. (1972). Likelihood: An Account of the Statistical Concept of Likelihood and its Application to Scientific Inference. Cambridge: Cambridge University Press.

Feldberg, K., Schneider, H., Stadler, T., Schäfer-Verwimp, A., Schmidt, A. R., and Heinrichs, J. (2014). Epiphytic leafy liverworts diversified in angiospermdominated forests. Sci. Rep. 4:5974. doi: 10.1038/srep05974

Fisher, G. C. (1914). Seed development in the genus Peperomia. Bull. Torrey Bot. Club 41, 137-156.

FitzJohn, R. G. (2012). Diversitree: comparative phylogenetic analyses of diversification in R. Methods Ecol. Evol. 3, 1084-1092. doi: 10.1111/j.2041210X.2012.00234.X

Frenzke, L., Lederer, A., Malanin, M., Eichhorn, K. -J., Neinhuis, C., and Voigt, D. (2016). Plant pressure sensitive adhesives: similar chemical properties in distantly related plant lineages. Planta 244, 145-154. doi: 10.1007/s00425-0162496-4

Frenzke, L., Scheiris, E., Pino, G., Symmank, L., Goetghebeur, P., Neinhuis, et al. (2015). A revised infrageneric classification of the genus Peperomia Ruiz \& Pav. (Piperaceae). Taxon 64, 424-444. doi: 10.12705/643.4

Frodin, D. G. (2004). History and concepts of big plant genera. Taxon 53, 753-776. doi: $10.2307 / 4135449$

Gentry, A. H., and Dodson, C. H. (1987). Diversity and biogeography of neotropical vascular epiphytes. Ann. Missouri Bot. Gard. 74, 205-233.

Givnish, T. J., Barfuss, M. H. J., Ee, B. V., Riina, R., Schulte, K., Horres, R., Gonsiska, P. A., et al. (2014). Adaptive radiation, correlated and contingent evolution, and net species diversification in Bromeliaceae. Mol. Phylogenet. Evol. 71, 55-78. doi: 10.1016/j.ympev.2013.10.010

Givnish, T. J., Spalink, D., Ames, M., Lyon, S. P., Hunter, S. J., Zuluaga, A., et al. (2015). Orchid phylogenomics and multiple drivers of their extraordinary diversification. Proc. R. Soc. Lond. B 282:1814. doi: 10.1098/rspb. 2015.1553

Granados Mendoza, C., Naumann, J., Samain, M. S., Goetghebeur, P., De Smet, Y., and Wanke, S. (2015). A genome-scale mining strategy for recovering novel rapidly-evolving nuclear single-copy genes for addressing shallow-scale phylogenetics in Hydrangea. BMC Evol. Biol. 15:132. doi: 10.1186/s12862-0150416-Z

Gravendeel, B., Smithson, A., Slik, F. J. W., and Schuiteman, A. (2004). Epiphytism and pollinator specialization: drivers for orchid diversity? Philos. Trans. R. Soc. B 359, 1523-1535. doi: 10.1098/rstb.2004.1529

Guindon, S., and Gascuel, O. (2003). A simple, fast and accurate method to estimate large phylogenies by maximum likelihood. Syst. Biol. 52, 696-704. doi: 10.1080/10635150390235520

Hughes, L., Dunlop, M., French, K., Leishman, M. R., Rice, B., Rodgerson, L., et al. (1994). Predicting dispersal spectra: a minimal set of hypotheses based on plant attributes. J. Ecol. 82, 933-950. doi: 10.2307/2261456

Isnard, S., Prosperi, J., Wanke, S., Wagner, S. T., Samain, M. -S., Trueba, S., et al. (2012). Growth form evolution in Piperales and its relevance for understanding angiosperm diversification: an integrative approach combining plant architecture, anatomy, and biomechanics. Int. J. Plant Sci. 173, 610-639. doi: $10.1086 / 665821$

Johnson, D. S. (1900). On the endosperm and embryo of Peperomia pellucida. Bot. Gaz. 30, 1-11.

Krömer, T., Kessler, M., and Gradstein, S. R. (2007). Vertical stratification of vascular epiphytes in submontane and montane forest of the Bolivian Andes: the importance of the understory. Plant Ecol. 189, 261-278. doi: 10.1007/s11258-006-9182-8

Kubitzki, K., Rohwer, J. G., and Bittrich, V. (1993). Flowering Plants, Dicotyledons: Magnoliid, Hamamelid, and Caryophyllid Families. New York, NY: Springer.

Maddison, W. P., Midford, P. E., and Otto, S. P. (2007). Estimating a binary character's effect on speciation and extinction. Syst. Biol. 56, 701-710. doi: 10.1080/10635150701607033

Madison, M. (1979). Additional observations on ant-gardens in Amazonas. Selbyana 5, 107-115.

Martínez-Colín, M. A., Engleman, E. M., and Koch, S. D. (2006). Contribución al conocimiento de Peperomia (Piperaceae): fruto y semilla. Bol. Soc. Bot. Méx. 78, 83-94.

Mathieu, G., Samain, M.-S., Reynders, M., and Goetghebeur, P. (2008). Taxonomy of the Peperomia species (Piperaceae) with pseudo-epiphyllous inflorescences, including four new species. Bot. J. Linn. Soc. 157, 177-196. doi: 10.1111/j.10958339.2008.00777.x

Mathieu, G., Symmank, L., Callejas, R., Wanke, S., Neinhuis, C., Goetghebeur, P., et al. (2011). New geophytic Peperomia (Piperaceae) species from Mexico, Belize and Costa Rica. Rev. Mex. Biodivers. 82, 357-382.

Melcher, I. M., Bouman, F., and Cleef, A. (2000). Seed dispersal in Páramo plants: epizoochorous and hydrochorous taxa. Plant Biol. 2, 40-52. doi: 10.1055/s2000-9146

Midford, P., and Maddison, W. (2006). Correl Package for Mesquite v. 0.1. Available at: http://mesquiteproject.org

Müller, K., Quandt, D., Müller, J., and Neinhuis, C. (2010). PhyDE-1: Phylogenetic Data Editor. V. 0.9971. Available at: http://www.phyde.de

Naumann, J., Symmank, L., Samain, M.-S., Müller, K. F., Neinhuis, C., dePamphilis, C. W., et al. (2011). Chasing the hare - evaluating the phylogenetic utility of a nuclear single copy gene region at and below species level within the species rich group Peperomia (Piperaceae). BMC Evol. Biol. 11:357. doi: 10.1186/14712148-11-357

Neal, R. M. (2003). Slice sampling. Ann. Stat. 31, 705-767.

Ng, J., and Smith, S. D. (2014). How traits shape trees: new approaches for detecting character state-dependent lineage diversification. J. Evol. Biol. 27, 2035-2045. doi: $10.1111 /$ jeb.12460

O'Meara, B. C. (2012). Evolutionary inferences from phylogenies: a review of methods. Annu. Rev. Ecol. Evol. Syst. 43, 267-285. doi: 10.1146/annurevecolsys-110411-160331

Pagel, M. (1994). Detecting correlated evolution on phylogenies: a general method for the comparative analysis of discrete characters. Proc. R. Soc. Lond. B. 255, 37-45. doi: 10.1098/rspb.1994.0006

Paradis, E., Claude, J., and Strimmer, K. (2004). APE: analyses of phylogenetics and evolution in R language. Bioinformatics 20, 289-290. doi: 10.1093/bioinformatics/btg412

Pino, G., Cieza, N., Wanke, S., and Samain, M. -S. (2012). New succulent window-leaved Peperomias from Peru. Haseltonia 18, 3-26. doi: 10.2985/026. 018.0102 
Plummer, M., Best, N., Cowles, K., and Vines, K. (2006). CODA: convergence diagnosis and output analysis for MCMC. R News 6, 7-11.

Posada, D. (2008). jModelTest: phylogenetic model averaging. Mol. Biol. Evol. 25, 1253-1256. doi: 10.1093/molbev/msn083

Rabosky, D. L. (2010). Extinction rates should not be estimated from molecular phylogenies. Evolution 64, 1816-1824. doi: 10.1111/j.1558-5646.2009.00926.x

Rabosky, D. L. (2014). Automatic detection of key innovations, rate shifts, and diversity-dependence on phylogenetic trees. PLOS ONE 9:e89543. doi: 10.1371/journal.pone.0089543

Rabosky, D. L., Donnellan, S. C., Talaba, A. L., and Lovette, I. J. (2007). Exceptional among-lineage variation in diversification rates during the radiation of Australia's most diverse vertebrate clade. Proc. R. Soc. B Biol. Sci. 274, 29152923. doi: 10.1098/rspb.2007.0924

Rabosky, D. L., and Goldberg, E. E. (2015). Model inadequacy and mistaken inferences of trait-dependent speciation. Syst. Biol. 64, 340-355. doi: 10.1093/sysbio/syu131

Rabosky, D. L., Grundler, M., Anderson, C., Shi, J. J., and Brown, J. W., Huang, H., et al. (2014). BAMMtools: an R package for the analysis of evolutionary dynamics on phylogenetic trees. Methods Ecol. Evol. 5, 701-707. doi: 10.1111/2041-210X.12199

Rambaut, A. (2008). FigTree Version 1.4.0. Available at: http://tree.bio.ed.ac.uk/so ftware/figtree

Rambaut, A., and Drummond, A. J. (2009). Tracer Version. 1.5. Available at: http://beast.bio.ed.ac.uk/Tracer

Rauh, W. (1950). Morphologie der Nutzpflanzen. Leipzig: Quelle \& Meyer.

Ridley, H. N. (1930). The Dispersal of Plants Throughout the World. Ashford: Reeve.

Ronquist, F., and Huelsenbeck, J. P. (2003). MrBayes 3: bayesian phylogenetic inference under mixed models. Bioinformatics 19, 1572-1574. doi: 10.1093/bioinformatics/btg180

Samain, M. -S., Mathieu, G., Pino, G., Symmank, L., Cieza, N., Neinhuis, C., et al. (2011). The geophytic Peperomia subgenus Tildenia (Piperaceae) in the Andes with the description of new species in a phylogenetic framework. Plant Ecol. Evol. 144, 148-176. doi: 10.5091/plecevo.2011.470

Samain, M. -S., Mathieu, G., Vanderschaeve, L., Wanke, S., Neinhuis, C., and Goetghebeur, P. (2007). Nomenclature and typification of subdivisional names in the genus Peperomia (Piperaceae). Taxon 56, 229-236. doi: 10.2307/25065756

Samain, M.-S., Mathieu, G., Wanke, S., Neinhuis, C., and Goetghebeur, P. (2008). Verhuellia revisited - unravelling its intricate taxonomic history and a new subfamilial classification of Piperaceae. Taxon 57, 583-587. doi: $10.2307 / 25066024$

Samain, M. -S., Vanderschaeve, L., Chaerle, P., Goetghebeur, P., Neinhuis, C., and Wanke, S. (2009). Is morphology telling the truth about the evolution of the species rich genus Peperomia (Piperaceae)? Plant Syst. Evol. 278, 1-21. doi: 10.1007/s00606-008-0113-0

Sanderson, M. J. (2002). Estimating absolute rates of molecular evolution and divergence times: a penalized likelihood approach. Mol. Biol. Evol. 19, 101-109.

Sanderson, M. J., and Donoghue, M. J. (1994). Shifts in diversification rate with the origin of angiosperms. Science 264, 1590-1593.

Schluter, D., Price, T., Mooers, A. Ø., and Ludwig, D. (1997). Likelihood of ancestor states in adaptive radiation. Evolution 51:1699. doi: 10.2307/2410994

Silvera, K., Santiago, L. S., Cushman, J. C., and Winter, K. (2009). Crassulacean acid metabolism and epiphytism linked to adaptive radiations in the Orchidaceae. Plant Physiol. 149, 1838-1847. doi: 10.1104/pp.108.132555

Smith, J. F., Stevens, A. C., Tepe, E. J., and Davidson, C. (2008). Placing the origin of two species-rich genera in the late cretaceous with later species divergence in the tertiary: a phylogenetic, biogeographic and molecular dating analysis of Piper and Peperomia (Piperaceae). Plant Syst. Evol. 275, 9-30. doi: 10.1007/s00606-008-0056-5

Sorensen, A. E. (1986). Seed dispersal by adhesion. Annu. Rev. Ecol. Evol. Syst. 17, $443-463$.
Stamatakis, A., Ludwig, T., and Meier, H. (2005). RAxML-III: a fast program for maximum likelihood-based inference of large phylogenetic trees. Bioinforma. Oxf. Engl. 21, 456-463. doi: 10.1093/bioinformatics/bti191

Symmank, L., Samain, M.-S., Goetghebeur, P., Mathieu, G., Neinhuis, C., and Wanke, S. (2008). "Independent evolution of different terrestrial life forms in the genus Peperomia (Piperaceae) and implications for biogeography of the tuberous species," in Systematics 2008, eds S. R. Gradstein, S. Klatt, F. Normann, P. Weigelt, R. Willmann, and R. Wilson (Göttingen: Universitätsverlag Göttingen).

Symmank, L., Samain, M.-S., Smith, J. F., Pino, G., Stoll, A., Goetghebeur, P., et al. (2011). The extraordinary journey of Peperomia subgenus Tildenia (Piperaceae): insights into diversification and colonization patterns from its cradle in Peru to the Trans-Mexican Volcanic Belt. J. Biogeog. 38, 2337-2349. doi: 10.1111/j.1365-2699.2011.02586.x

Trelease, W., and Yuncker, T. G. (1950). The Piperaceae of Northern South America. Urbana, IL: University of Illinois Press.

Valdebenito, H. A., Stuessy, T. F., and Crawford, D. J. (1990). Synonymy in Peperomia berteroana (Piperaceae) results in biological disjunction between Pacific and Atlantic Oceans. Brittonia 42, 121-124. doi: 10.2307/28 07626

Valdebenito, H. A., Stuessy, T. F., Crawford, D. J., and Silva, O. M. (1992). Evolution of Peperomia (Piperaceae) in the Juan Fernandez Islands, Chile. Plant Syst. Evol. 182, 107-119. doi: 10.1007/BF00941416

van der Pijl, L. (1982). Principles of Dispersal in Higher Plants. New York, NY: Springer.

Wanke, S., Jaramillo, M. A., Borsch, T., Samain, M. -S., Quandt, D., and Neinhuis, C. (2007a). Evolution of Piperales-matK gene and trnK intron sequence data reveal lineage specific resolution contrast. Mol. Phylogenet. Evol. 42, 477-497. doi: 10.1016/j.ympev.2006.07.007

Wanke, S., Samain, M.-S., Vanderschaeve, L., Mathieu, G., Goetghebeur, P., and Neinhuis, C. (2006). Phylogeny of the genus Peperomia (Piperaceae) inferred from the trnK/matK region (cpDNA). Plant Biol. 8, 93-102. doi: 10.1055/s2005-873060

Wanke, S., Vanderschaeve, L., Mathieu, G., Neinhuis, C., Goetghebeur, P., and Samain, M. -S. (2007b). From forgotten taxon to a missing link? The position of the genus Verhuellia (Piperaceae) revealed by molecules. Ann. Bot. 99, 1231-1238. doi: 10.1093/aob/mcm063

Willson, M., and Traveset, A. (1990). “The ecology of seed dispersal," in Seeds: The Ecology of Regeneration in Plant Communities, 2nd Edn, ed. M. Fenner (Wallingford: CABI Publishing), 85-110.

Yuncker, T. G. (1953). The piperaceae of Argentina, Bolivia and Chile. Lilloa 27, 97-303.

Yuncker, T. G. (1958). The piperaceae: a family profile. Brittonia 10, 1-7.

Yuncker, T. G. (1974). The piperaceae of brasil III. Peperomia: taxa of uncertain status. Hoehnea 4, 71-413.

Zotz, G. (2013). The systematic distribution of vascular epiphytes a critical update. Bot. J. Linn. Soc. 171, 453-481. doi: 10.1111/boj. 12010

Conflict of Interest Statement: The authors declare that the research was conducted in the absence of any commercial or financial relationships that could be construed as a potential conflict of interest.

Copyright (c) 2016 Frenzke, Goetghebeur, Neinhuis, Samain and Wanke. This is an open-access article distributed under the terms of the Creative Commons Attribution License (CC BY). The use, distribution or reproduction in other forums is permitted, provided the original author(s) or licensor are credited and that the original publication in this journal is cited, in accordance with accepted academic practice. No use, distribution or reproduction is permitted which does not comply with these terms. 